\begin{tabular}{|c|l|}
\hline Title & Multi-objective topology optimization of magnetic couplers for wireless power transfer \\
\hline Author(s) & Gong, Y unyi; Otomo, Y oshitsugu; Igarashi, Hajime \\
\hline Citation & $\begin{array}{l}\text { International Journal of A pplied Electromagnetics and Mechanics, 64(1-4), 325-333 } \\
\text { https://doi.org/L0.3233/AA E-209337 }\end{array}$ \\
\hline Issue Date & 2020-12-10 \\
\hline Doc URL & http://hdl.handle.net/2115/80240 \\
\hline Rights & The final publication is available at IOS Press through http://dx.doi.org/10.3233/AAE-209337 \\
\hline Type & article (author version) \\
\hline File Information & Multi-Objective Topology Optimization of Magnetic Couplers for Wireless Power T ransfer.pdf \\
\hline
\end{tabular}

Instructions for use 


\title{
Multi-Objective Topology Optimization of Magnetic Couplers for Wireless Power Transfer
}

\author{
Yunyi Gong ${ }^{a,{ }^{*}}$, Yoshitsugu Otomo ${ }^{a}$ and Hajime Igarashi ${ }^{\text {a }}$ \\ ${ }^{a}$ Graduate School of information Science and Technology, \\ Hokkaido University, 060-0814, Sapporo, Japan
}

\begin{abstract}
In this paper, the multi-objective topology optimizations of wireless power transfer (WPT) devices with two different coil geometries are proposed for obtaining the designs with good balance between transfer efficiency and safety. For this purpose, the proposed method adopts the normalized Gaussian network (NGnet) and Non-dominated Sorting Genetic Algorithm II (NSGA-II). In addition, the optimization under the different constraint on ferrite volume is carried out to verify its influence on optimization results. It has been shown that the proposed method successfully provides the Pareto solution to the design problem of the WPT device.
\end{abstract}

Keywords: wireless power transfer, topology optimization, Pareto solution, electric vehicles.

\section{Introduction}

Recently, the increasing attention on electric vehicles (EV) brought by concerns of the global warming and air pollution has stimulated the research of wireless power transfer (WPT) which is expected to realize more reliable and safer charging systems for EV. Over the years, several couplers for WPT including circular [1] and double D [2] shapes have been proposed. So far many studies for optimizing structure and design parameters, e.g, for circular pad in [3], and for double D in [4] have been made. Moreover, in [3], the effect of the magnetic core configuration on the performance has been discussed.

In addition to the coupling efficiency, the safety of WPT devices is also a focus of WPT researches. Limitation of the magnetic flux exposure is typically imposed to ensure the safety of electromagnetic instruments. As defined in [5] by ICNIRP, for magnetic field frequencies ranging from $3 \mathrm{kHz}$ to 10 $\mathrm{MHz}$, the flux exposure to human bodies should be limited up to $27 \mu \mathrm{T}$ for general public exposure, and $100 \mu \mathrm{T}$ for occupational exposure.

In [4] and [6], to ensure the safety of WPT system, the multi-objective optimization of the WPT couplers with respect to the coupling coefficient and leakage magnetic flux density has been reported. In those works, the WPT structure is designed using the parameter optimization for which, however, the choice of the design parameters gives significant influence on the optimization result and performance. In contrast, the topology optimization does not need to introduce the design parameter. In particular, the on/off method based on the normalized Gaussian network (NGnet) has been proved effective for the optimization of rotating machines [7] and antennas [8,9]. This method has also successfully applied to the topology optimization of a WPT device with single objective function [10].

In this work, a multi-objective topology optimization, employing NGnet for the topology representation and Non-dominated Sorting Genetic Algorithm II (NSGA-II) for the optimizer, has been firstly applied to the design of the ferrite core in the coupler of a WPT device, seeking for a good balance between the coupling efficiency and amount of leakage fields.

\section{Coil geometries}

Besides the basic circular coil, a lot of different coil geometries has been proposed, based on the consideration of the transfer efficiency, tolerance to position misalignment and etc. [2]. In this work, we focus on the two different coil systems whose their design concept will be explained below.

A circular coupler pad with magnetic cores in bar-shape is shown in Fig. 1 (a). Although the circular 
pattern has been studied as one of the promising WPTs for a long time, some limits have been pointed out at the same time, including the coupling limitation at certain coil diameters [2]. To overcome the limitation of the circular coil, the double D (DD) structure, which is illustrated in Fig. 1 (b), has been proposed [2]. In this WPT device, two coplanar coils are placed above bar ferrite, with equal and opposite current flowing in the two coils, respectively.

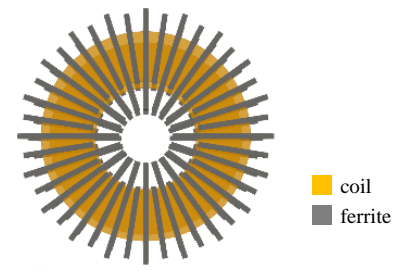

(a) Coupler with double circular coil

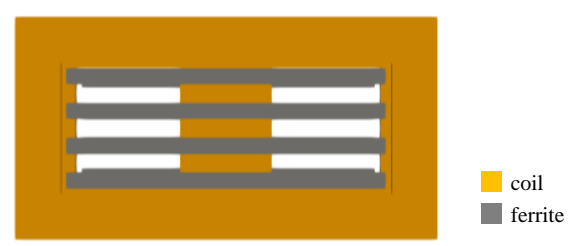

(b) Coupler with double D coil

Fig. 1. Coupler with two coil geometries (top view)

\section{Normalized Gaussian Network}

In this work, the topology and shape of the ferrite core is represented using the normalized Gaussian network (NGnet) [7], which defines the binary state \{on, off \} for each finite element. In NGnet, the state $V_{e}$ which corresponds to the material attribute (ferrite/air) of a finite element is determined from

$$
V_{e}= \begin{cases}\text { on } & \phi\left(x_{\boldsymbol{e}}\right) \geq 0 \\ \text { off } & \phi\left(\boldsymbol{x}_{\boldsymbol{e}}\right) \leq 0\end{cases}
$$

where $\boldsymbol{x}_{\boldsymbol{e}}$ and $\phi$ denote the element center and the shape function defined by

$$
\phi\left(\boldsymbol{x}_{\boldsymbol{e}}\right)=\sum_{j=1}^{N_{g}} w_{i} b_{i}(x)
$$

In equation (2), $N_{g}, w_{i}$ and $b_{i}$ denote the number of basis functions, weighting coefficient and normalized Gaussian function given by

$$
\begin{gathered}
b_{i}=\frac{G_{i}(\boldsymbol{x})}{\sum_{j=1}^{N g} G_{j}(\boldsymbol{x})} \\
G_{i}(\boldsymbol{x})=\frac{1}{2 \pi \sigma^{2}} \exp \left(-\frac{1}{2 \sigma^{2}}\left|\boldsymbol{x}-\boldsymbol{\mu}_{\boldsymbol{i}}\right|^{2}\right)
\end{gathered}
$$

where $\boldsymbol{\mu}_{\boldsymbol{i}}$ and $\sigma$ denote the center of the $i$-th Gaussian function and the standard deviation, respectively. The material attribute is set to ferrite (air) when $\phi\left(\boldsymbol{x}_{\boldsymbol{e}}\right) \geq 0\left(\phi\left(\boldsymbol{x}_{\boldsymbol{e}}\right) \leq 0\right)$. The ferrite shape is determined from the weights $w_{i}, i=1,2, \ldots, N_{g}$. This means that the topology optimization is reduced to the parameter optimization with respect to $w_{i}$. The process of the topology optimization based on NGnet is illustrated in Fig. 2.

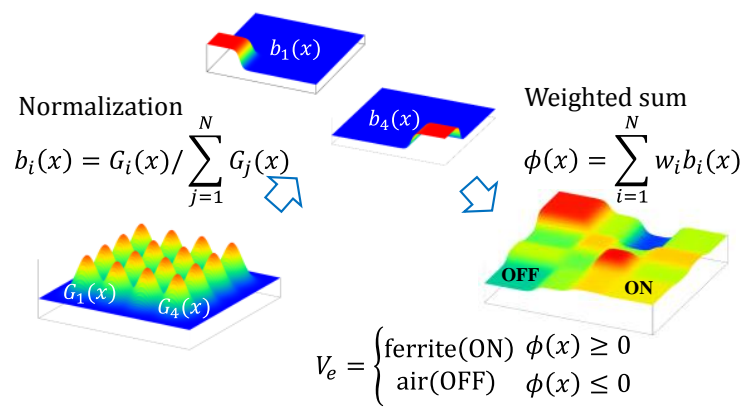

Fig. 2. Process of NGnet calculation

\section{Non-dominated Sorting Genetic Algorithm II}

In this work, Non-dominated Sorting Genetic Algorithm II (NSGA-II) [11] is adopted in order to solve the multi-objective optimization problem. Thanks to NSGA-II, we can obtain well spread Pareto 
solutions which can have a non-convex distribution. As shown in Fig. 3, the process of NSGA-II is based on the algorithm that is extended from the conventional genetic algorithm, except that the nondominated sorting is introduced instead of the normal sorting, which gives the ranks to the individuals with respect to the Pareto dominance. As a result of NSGA-II, Pareto front is obtained, which contains all the individuals who are not dominated by the others.

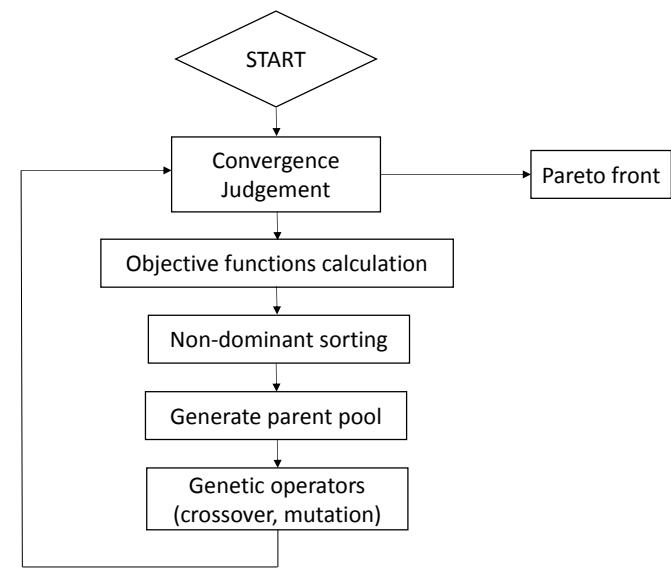

Fig. 3. Process of NSGA-II

\section{Design model and Optimization problem}

The design model and its size are shown in Fig. 4 (a). The optimal ferrite shape is determined in the design region, while the leakage flux is evaluated in the evaluation region. In Fig. 4 (b), there are air gaps of $4 \mathrm{~mm}$ between the coil and ferrite core, which is expected to increase the coupling coefficient, according to [12]. For reducing the computation cost, we consider only the $1 / 4$ model of the whole coupler for the optimization in which 69 Gaussian functions are uniformly deployed, as shown in Fig. 5. From the optimization result, the whole structure is built according to the symmetry. The design model and Gaussians arrangement of coupler for the double D coil are shown in Figs. 6 and 7, where 126 Gaussians are arranged uniformly for the 1/4 model in a similar way.

To compute the coupling coefficient and leakage magnetic field, we use an in-house 3D finite element code for magnetostatic fields whose unknown is the line integral of the vector potential along element edges. For analysis of both couplers, the boundary condition is imposed on the model boundaries which is $1000 \mathrm{~mm}$ away from the couplers.

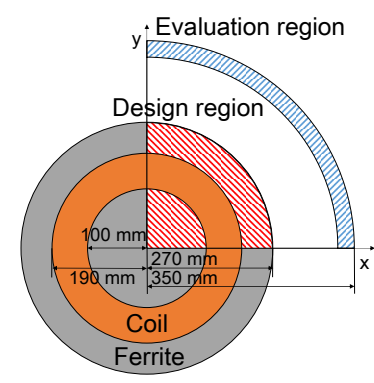

(a) Top view

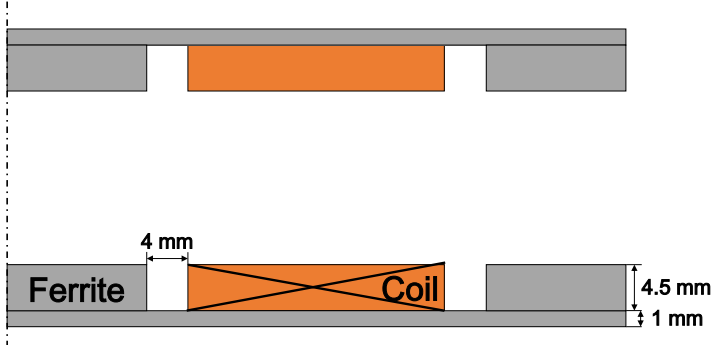

(b) Front view

Fig. 4. Design model of coupler with Circular coil

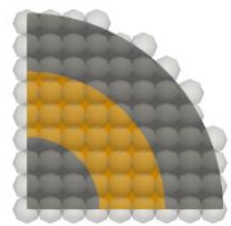

Fig. 5. Arrangement of Gaussian functions for Circular coupler.

The circles represent the contour lines the distributed Gaussian functions with the value of $\sigma$. 


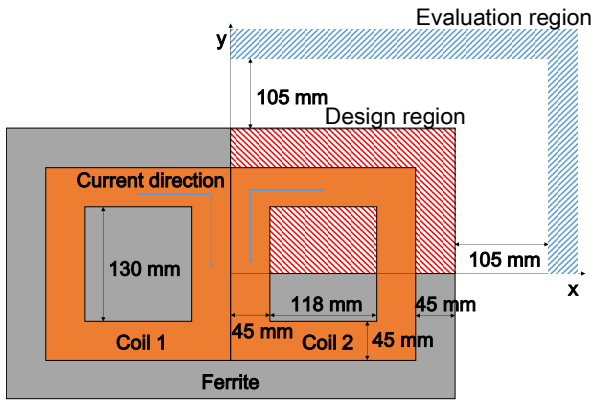

(a) Top view

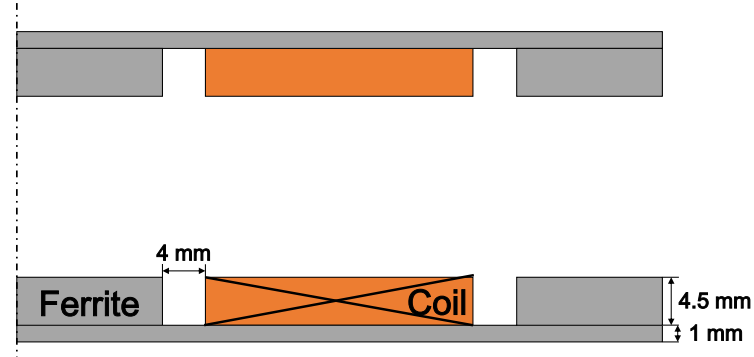

(b) Front view

Fig. 6. Design model of coupler with Double D coil

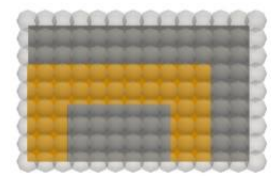

Fig. 7. Arrangement of Gaussian functions for Double D coupler

The optimization problem is defined by

$$
F=\left\{\begin{array}{c}
k \rightarrow \text { max. } \\
B_{\max } \rightarrow \text { min. }
\end{array} \text {, sub. to } \Omega_{I} \leq c * \Omega_{R}\right.
$$

where $k$ and $B_{\max }$ are the coupling coefficient and largest flux in evaluation region which represents the leakage flux, and $\Omega_{I}$ and $\Omega_{R}$ are the ferrite-core volume of the individuals and reference model respectively, while $c$ is a constant coefficient taking 1.0 or 1.1. The volume constraint is introduced to suppress the volume as priority when $c=1$, and to obtain better performance when $c=1.1$.

The weighting coefficients of all the Gaussians are treated as the gene in NSGA-II whose procedure ends at the 150th generation. In each generation, 120 individuals sorted with respect to the fitness are chosen as the parents. After the crossover between the parents and the mutation is applied to the individuals according to given probability, and 120 children are generated. The parameters of WPT system and NSGA-II are summarized in Table 1.

Table 1

Parameters of WPT and NSGA-II

\begin{tabular}{ll|ll}
\hline Parameter & Value & Parameter & Value \\
\hline$I$ (abs) & $23 \mathrm{~A}$ & Parent population & 120 \\
Transmission distance & $100 \mathrm{~mm}$ & Child population & 120 \\
$f$ & $20 \mathrm{kHz}$ & End generation & 150 \\
Turns of coil & $20($ Circular), 10 (DD) & Crossover probability & $95 \%$ \\
Ferrite $\mu_{r}$ & 1000 & Mutation probability & $5 \%$ \\
\hline
\end{tabular}

\section{Optimization results}

\subsection{Circular coil}

The Pareto front at the 150-th generation for the circular coil is shown in Fig. 8, where the red point represents the reference model, while the circles and triangles represent the optimized models under constraints whose constant coefficient $c$ are 1.0 and 1.1 respectively.

As expected, the trade-off relationship between the coupling efficiency and leakage field can be observed. Although there is no solution which has a better coupling efficiency compared to the reference model, all of them have a much lower magnetic leakage flux compared to the reference model. When $c$ is increased from 1.0 to 1.1 , both performances are a bit improved, whereas the coupling efficiency is still lower than that of the reference model. This suggests that NGnet could not well represent the barshaped ferrite which has a fine structure. 


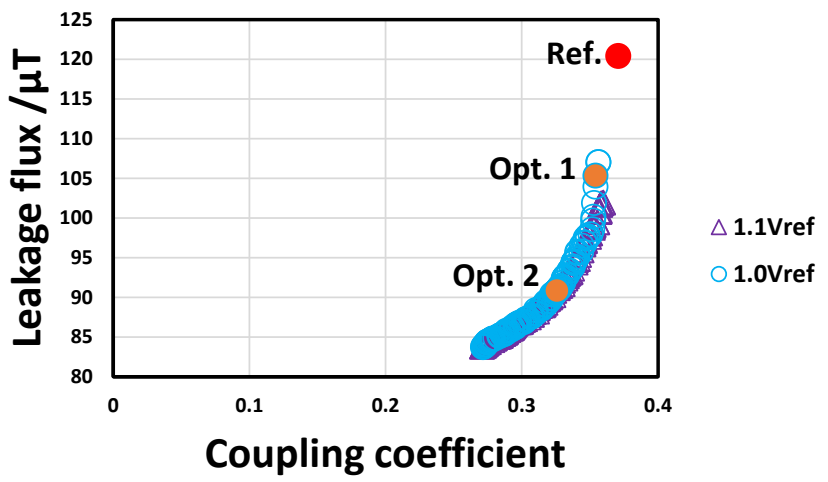

Fig. 8. Pareto font of optimization for circular coupler

The reference model and two typical optimized solutions marked by the orange points in Fig. 8 are illustrated in Fig.9 with the magnetic flux distribution. As can be seen in Fig. 9, the proposed method resulted in the non-separated ferrite cores with holes, which might be hardly obtained by the conventional parameter optimization. There are the significant differences among the shapes of the reference and optimized ferrite cores.
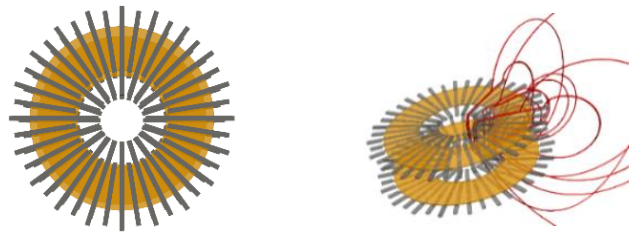

(a) Reference model $(k=37.1 \%, B=120.4 \mu \mathrm{T})$
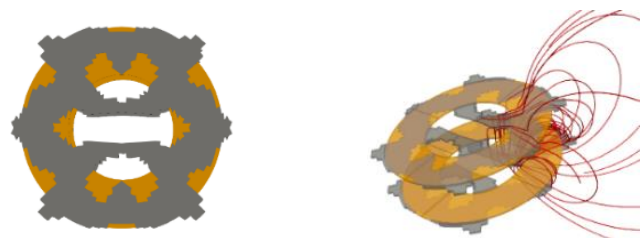

(b) Optmized model $1(k=35.4 \%, B=105.3 \mu \mathrm{T})$
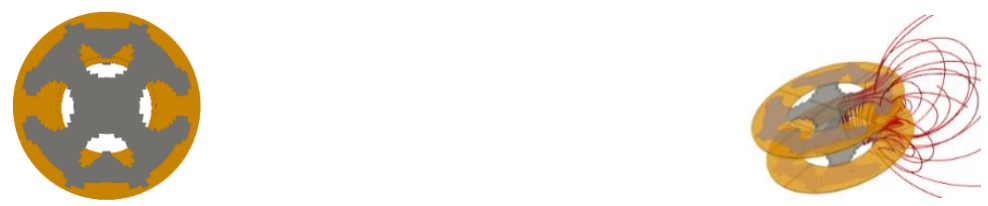

(c) Optmized model $2(k=32.6 \%, B=90.8 \mu \mathrm{T})$

Fig. 9. Reference model and optimized models (Circular)

\subsection{Double D coil}

As for the couplers with the DD coils, the Pareto solutions shown in Fig.10 are obtained, while the difference between reference and optimized models is smaller than that for the circular-coil model. Increase in $c$ from 1.0 to 1.1 results in a small improvement of the performance again.

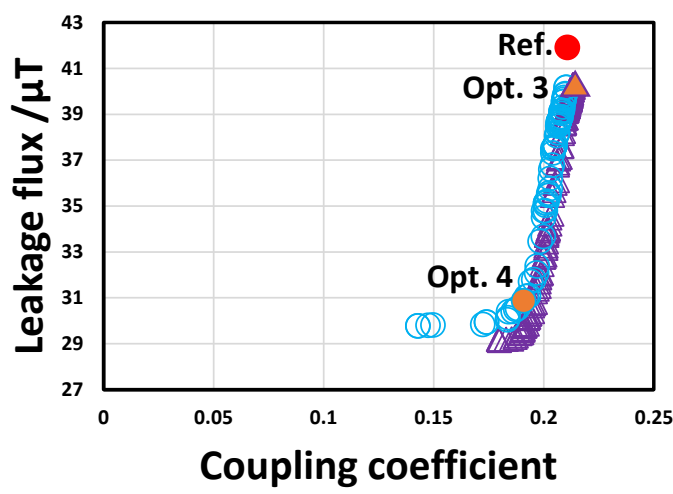

$\triangle 1.1$ Vref

1.0Vref

Fig. 10. Pareto font of optimization for DD coupler

The reference model and representative optimized models are shown in Fig. 11. Both optimized results 
include the slender ferrite core whose width decreases outside, which would reduce the leakage magnetic fields. Moreover, the proposed optimization method results in the non-separated ferrite cores also in this case. The spatial resolution of NGnet would be insufficient for the expression of the bar ferrite in the reference model. The higher resolution, which can be made by increasing the number of Gaussians, leads to, however, complicated core shapes. The proposed method can decrease the leakage flux while it is again difficult to make the coupling efficiency greater than that of the reference model. This remains as the future problem.
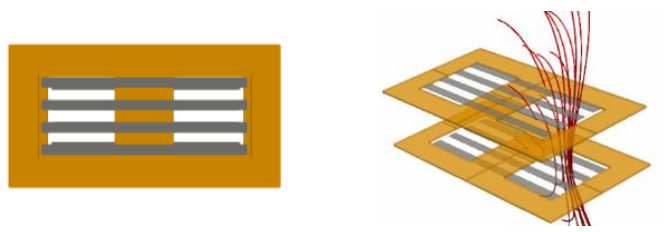

(a) Reference model $(k=21.1 \%, B=42.0 \mu \mathrm{T})$
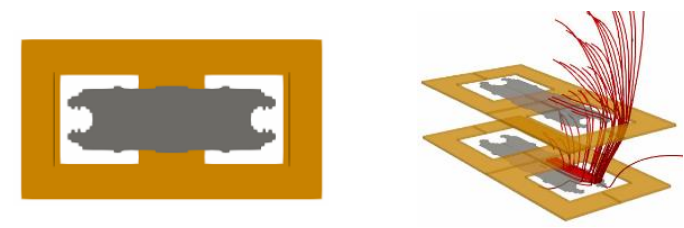

(b) Optmized model $3(k=21.3 \%, B=40.3 \mu \mathrm{T})$
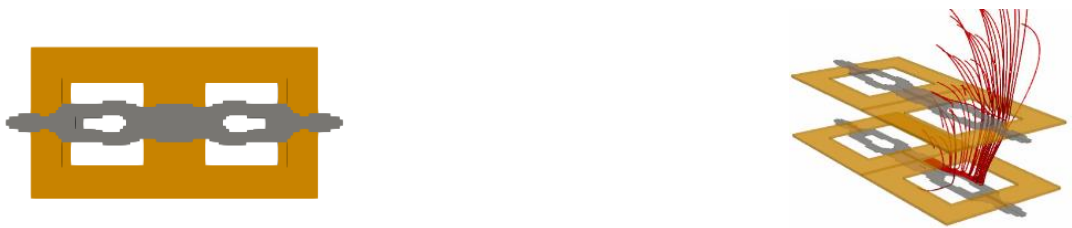

(c) Optmized model $4(k=19.1 \%, B=30.9 \mu \mathrm{T})$

Fig. 11. Reference model and optimized models (DD)

\section{Conclusion}

In this paper, a multi-objective topology optimization with respect to the coupling coefficient and leakage magnetic flux has been applied to the design of the magnetic core of the circular and double D WPT couplers, under a given constraint to the ferrite volume. Using the NGnet method for representation of the ferrite core, and NSGA-II for the optimizer, the Pareto front has been successfully obtained. Although the coupling efficiency of the optimized couplers is lower than that of the reference models, the leakage flux is made smaller by the optimization. The improvement of the proposed method to obtain better coupling efficiency remains for the future work.

\section{References}

[1] X. Liu, S. Y. Hui, "Optimal design of a hybrid winding structure for planar contactless battery charging platform," IEEE Trans. Power Electronics, vol. 23, no. 1, pp. 455-463, 2008.

[2] M. Budhia, et al. "Development of a single-sided flux magnetic coupler for electric vehicle IPT charging systems." IEEE Transactions on Industrial Electronics, vol. 60, no. 1, pp. 318-328, 2013.

[3] M. Budhia, G. A. Covic, J. T. Boys, "Design and optimization of circular magnetic structures for lumped inductive power transfer systems," IEEE Trans. Power Electronics, vol. 26, no. 11, pp. 3096-3108, 2011.

[4] Z. Luo, X. Wei, G. A. Covic, "Multi-objective Optimization of Double D Coils for Wireless Charging System," 2018 IEEE International Power Electronics and Application Conference and Exposition (PEAC), pp 1-6, 2018.

[5] International Commission on Non-Ionizing Radiation Protection, "Guidelines for limiting exposure to time-varying electric and magnetic fields (1 Hz to $100 \mathrm{kHz}$ )," Health Phys., vol. 99, no. 6, pp. 818-836, 2010.

[6] T. Yilmaz, N. Hasan, R. Zane, et al, "Multi-objective optimization of circular magnetic couplers for wireless power transfer applications," IEEE Trans. Magn., vol. 53, no. 8, pp. 1-12, 2017.

[7] T. Sato, K. Watanabe, H. Igarashi, "Multimaterial topology optimization of electric machines based on normalized Gaussian network," IEEE Trans. Magn., vol.51, no.3, Art. no. 7202604, 2015.

[8] Y. Fangzhou, H. Igarashi, "Topology Optimization of Metamaterial Using Gaussian-Basis Functions," Journal of Advanced Simulation in Science and Engineering, vol.6, no.1, pp. 149-156, 2019.

[9] K. Itoh, H. Nakajima, H. Matsuda, M. Tanaka, H. Igarashi, "Development of Small Dielectric Lens for Slot Antenna Using Topology Optimization with Normalized Gaussian Network," IEICE Transactions on Electronics, vol. E101.C no.10, pp.784-790, 2018.

[10] Y. Otomo, H. Igarashi, “A 3-D Topology Optimization of Magnetic Cores for Wireless Power Transfer Device,” IEEE Trans. Magn., DOI: 10.1109/TMAG.2019.2900744, 2019.

[11] K. Deb, S. Agrawal, A. Pratap, et al, "A fast elitist non-dominated sorting genetic algorithm for multi-objective optimization: NSGA-II," International conference on parallel problem solving from nature, pp. 849-858, 2000.

[12] N. Hasan, T. Yilmaz, R. Zane, et al, "Multi-objective particle swarm optimization applied to the design of Wireless Power Transfer systems,” 2015 IEEE Wireless Power Transfer Conference (WPTC), pp 1-4, 2015. 